\title{
Targeted enhancement of flotillin- dependent endocytosis augments cellular uptake and impact of cytotoxic drugs
}

${\text { Farnaz Fekri }{ }^{1,2} \text {, John Abousawan }}^{1,2}$, Stephen Bautista ${ }^{1,2}$, Laura Orofiamma ${ }^{1,2}$, Roya M. Dayam ${ }^{1,2}$, Costin N. Antonescu $\mathbb{1}^{1,2,5^{*}}$ \& Raffi Karshafian $\mathbb{( \mathbb { D }}^{2,3,4,5^{*}}$

Cellular uptake is limiting for the efficacy of many cytotoxic drugs used to treat cancer. Identifying endocytic mechanisms that can be modulated with targeted, clinically-relevant interventions is important to enhance the efficacy of various cancer drugs. We identify that flotillin-dependent endocytosis can be targeted and upregulated by ultrasound and microbubble (USMB) treatments to enhance uptake and efficacy of cancer drugs such as cisplatin. USMB involves targeted ultrasound following administration of encapsulated microbubbles, used clinically for enhanced ultrasound image contrast. USMB treatments robustly enhanced internalization of the molecular scaffold protein flotillin, as well as flotillin-dependent fluid-phase internalization, a phenomenon dependent on the protein palmitoyltransferase DHHC5 and the Src-family kinase Fyn. USMB treatment enhanced DNA damage and cell killing elicited by the cytotoxic agent cisplatin in a flotillin-dependent manner. Thus, flotillin-dependent endocytosis can be modulated by clinically-relevant USMB treatments to enhance drug uptake and efficacy, revealing an important new strategy for targeted drug delivery for cancer treatment.

Conventional drug administration methods such as intravenous injection and oral administration rely largely on diffusion into tumors and cancer cells ${ }^{1}$. The action of many cytotoxic anti-cancer agents is thus limited by delivery of drug molecules across the plasma membrane of target cancer cells ${ }^{2,3}$. For example, cisplatin is effective in the treatment of various forms of cancer and functions by inducing DNA damage and defects in DNA replication once internalized into target cancer cells ${ }^{4,5}$. However, inefficient cisplatin uptake into cancer cells, toxicity to healthy tissues, and development of resistance limit the effectiveness of cisplatin in the clinic. Advanced drug delivery strategies that can target tumor tissues and efficiently enhance drug delivery to cancer cells can provide a better approach to overcome these limitations and thus improve the efficiency of therapeutic agents such as cisplatin.

The incorporation of drug molecules such as cisplatin into endocytic vesicles contributes to intracellular drug delivery ${ }^{6,7}$. There are several mechanistically distinct endocytic pathways that simultaneously operate within cells and which can be broadly categorized as either clathrin-dependent or clathrin-independent. Clathrin-mediated endocytosis is the principal route of internalization of receptor-bound macromolecules ${ }^{8}$. In contrast, clathrin-independent endocytosis (CIE) encompasses a number of distinct pathways that are diverse with respect to molecular machinery for cargo selection, endocytic vesicle formation and destination of internalized vesicles ${ }^{9-11}$. Several of these CIE pathways are high capacity and thus can mediate significant uptake of fluid-phase material ${ }^{9}$. Fluid-phase internalization is an attractive portal of entry for cancer drugs, as this can ensure the uptake of drug molecules without limitations imposed by specific molecular properties of these drugs (e.g. requirement to bind to specific cell-surface receptors). Therefore, the identification of fluid-phase endocytic

${ }^{1}$ Department of Chemistry and Biology, Ryerson University, Toronto, Canada. ${ }^{2}$ Graduate Program in Molecular Science, Ryerson University, Toronto, Ontario, M5B 2K3, Canada. ${ }^{3}$ Department of Physics, Ryerson University, Toronto, Ontario, M5B 2K3, Canada. ${ }^{4}$ Institute for Biomedical Engineering, Science and Technology (iBEST), a partnership between Ryerson University and St. Michael's Hospital, Toronto, Ontario, Canada. ${ }^{5}$ Keenan Research Centre for Biomedical Science of St. Michael's Hospital, Toronto, Ontario, Canada. *email: cantonescu@ryerson.ca; karshafian@ryerson.ca 
mechanisms that can be enhanced for drug delivery purposes and identifying therapeutically-compatible strategies to enhance such an endocytic pathway could provide avenues for achieving more efficient localized drug delivery to cancer cells.

An attractive CIE mechanism is one delineated by flotillin proteins. The flotillin family is composed of two highly homologous members: flotillin-1 (flot-1 or reggie-2) and flotillin-2 (flot- 2 or reggie-1) ${ }^{12}$. Both members of the family are ubiquitously expressed and highly conserved ${ }^{13,14}$. Flotillins exhibit cholesterol binding, hydrophobic hairpin insertion into lipid bilayers and acylation, and undergo homo- and hetero-oligomerization to form microdomains enriched in cholesterol and other specific lipids ${ }^{15-17}$. Flotillin microdomains can serve as scaffolding structures for signaling for a variety of cellular processes ${ }^{18}$ or to mediate a specific form of CIE ${ }^{9,10,19}$. Indeed this flotillin-dependent pathway can contribute substantially to fluid-phase endocytosis as well as the internalization of cargos such glycosylphosphatidylinositol (GPI)-linked proteins, cholera toxin B subunit, proteoglycans and their ligands and Niemann-Pick C1-like1 (NPC1L1) 10,20-24. Flotillin-dependent endocytosis can be modulated by certain cues such as EGF stimulation ${ }^{24}$.

Identifying cues and their signaling processes that can broadly enhance fluid-phase endocytosis, such as by enhancing flotillin-dependent endocytosis, would be very valuable from a cancer drug delivery perspective. Massive endocytosis (MEND) ${ }^{25-27}$ may be a particularly attractive mechanism for targeted drug delivery. MEND occurs in response to large intracellular $\mathrm{Ca}^{2+}$ transients (e.g. as occurs during plasma membrane perforations) and leads to large increases in fluid-phase endocytosis. While the molecular mechanisms underlying MEND remain incomplete, in fibroblasts MEND requires the enhanced activity of $\mathrm{DHHC}^{26}$, a member of the aspartate-histidine-histidine-cysteine (DHHC) palmitoyltransferase family ${ }^{28-30}$. Upon initiation of MEND, DHHC5 is thought to elicit the broad palmitoylation of cell surface proteins, which then triggers enhanced endocytosis through a poorly understood mechanism.

DHHC5 can palmitoylate flotillin- $2^{17}$ as well as neuronal proteins such as postsynaptic density-95 (PSD95), SynDIG1, GRIP1 and $\delta$-catenin, thus regulating membrane traffic of AMPA-type glutamate receptors in neurons ${ }^{31-34}$. DHHC5 activity and function is controlled by phosphorylation of DHHC5 on Y533, in a manner dependent on the Src-family kinase Fyn ${ }^{34}$, which in turn controls DHHC5 cell surface availability and function. Hence, dynamic control of palmitoylation by acyltransferases such as DHHC5 is an emerging signaling system that may be important to control fluid-phase endocytosis, either by direct control of endocytic proteins such as flotillins or by broad palmitoylation of many cell surface proteins (as thought to occur in MEND) to trigger fluid-phase endocytosis.

Leveraging the potential regulation of fluid-phase mechanisms, such as MEND-like processes, and/or flotillin-dependent endocytosis for drug delivery requires identification of treatments that can enhance the action of these endocytic processes. Ultrasound in combination with microbubbles (USMB) ${ }^{35-37}$ is an emerging strategy for targeted intracellular delivery of drug molecules ${ }^{38-41}$. Microbubbles (MBs) consist of a gas core with a diameter of less than $5 \mu \mathrm{m}$ that are encapsulated by lipids, albumin, or polymers, and are systemically injected into the vasculature for clinical ultrasound contrast enhancement ${ }^{42}$. In addition to this imaging phenomenon, the specific oscillatory response of microbubbles to ultrasound results in a multitude of highly localized effects on nearby cells and tissues ${ }^{43}$. Microbubbles in circulation are largely inert, and ultrasound can be focused to small $\left(\sim \mathrm{few} \mathrm{mm}^{3}\right)$ volumes, making USMB a very attractive strategy to achieve targeted effects in tissues.

USMB treatment elicits the formation of transient pores in the plasma membrane ${ }^{43}$. An emerging additional outcome of USMB treatment is an increase in the rate of endocytosis ${ }^{43-46}$. Indeed, we previously reported that USMB enhances the rate of both clathrin-mediated endocytosis and fluid-phase uptake into cells by distinct signaling mechanisms ${ }^{47}$. However, the molecular mechanisms and intracellular signals by which USMB triggers enhanced fluid-phase endocytosis and whether this phenomenon can contribute to targeted drug delivery to control cancer cell survival had not been addressed and must be understood in order for this drug delivery strategy to move closer to clinical use. Here, we examine the role of flotillin in the enhanced fluid-phase endocytosis following USMB treatment. We uncovered that USMB treatment elicits a novel signaling pathway that utilizes Fyn and DHHC5 to boost flotillin-dependent endocytosis, thus enhancing fluid-phase endocytosis in several cell types. Importantly, having established this novel mechanism to enhance fluid-phase endocytosis, we uncovered that the enhancement of flotillin-dependent endocytosis by USMB leads to enhanced killing of MDA-MB-231 triple-negative breast cancer cells by cisplatin, highlighting the potential clinical relevance of USMB in targeted drug delivery for cancer treatment by control of flotillin endocytosis.

\section{Results}

Ultrasound microbubble treatment triggers flotillin-dependent fluid-phase endocytosis. We previously reported that the acid sphingomyelinase (ASMase) inhibitor, desipramine, synergizes with USMB to enhance fluid-phase endocytosis, yet we excluded ASMase as a target for desipramine in the control of endocytosis upon USMB treatment ${ }^{47}$. Since desipramine is a clinically-approved drug, the possibility of a therapeutic combination of desipramine and USMB could be very promising, so we based our study here of the effects of USMB treatment on fluid-phase endocytosis on the combined use of USMB and desipramine.

We first investigated the contribution of flotillin to USMB-induced fluid-phase uptake. To do so, we tracked the internalization of the fluid-phase marker dextran conjugated to Alexa-488 (A488-dextran) for $30 \mathrm{~min}$ after USMB treatment in ARPE-19 (henceforth RPE) cells treated with siRNA to silence flotillin-1 and -2 (Fig. S2A). RPE cells are ideal to resolve the molecular mechanisms of cell surface phenomena such as endocytosis given their flat morphology. Consistent with our previous observations ${ }^{47}$, RPE cells transfected with non-targeting (control) siRNA exhibited $12.2 \pm 0.1 \%$ and $54.0 \pm 0.3 \%$ enhancements in fluid-phase endocytosis upon treatment with USMB or USMB + desipramine, respectively, compared to untreated (basal) cells ( $n=3, p<0.05$, Fig. 1A,B). As we observed previously ${ }^{47}$, desipramine treatment alone had no effect on fluid-phase internalization. Importantly, cells treated with flotillin siRNA exhibited no change in A488-dextran internalization upon treatment with either 
A

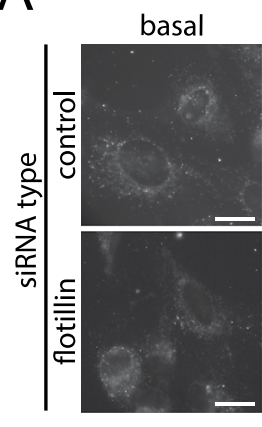

des

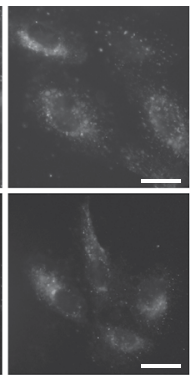

USMB

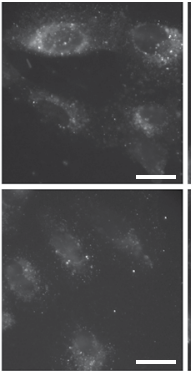

USMB+des

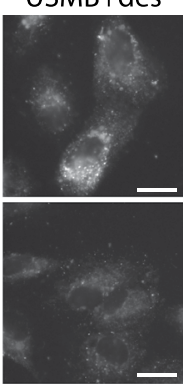

$B$

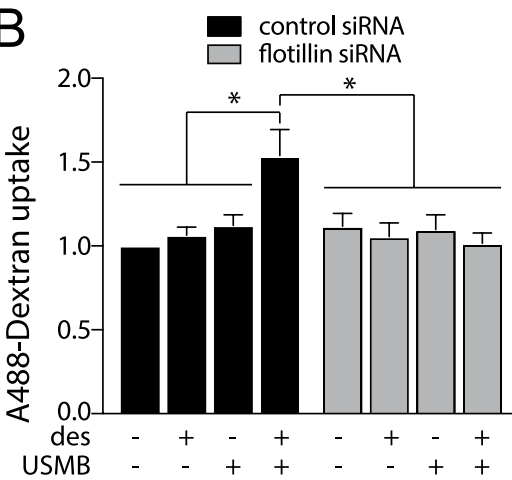

Figure 1. Flotillin is required for fluid-phase internalization triggered by USMB treatment. RPE cells were transfected with siRNA targeting flotillin-1 and -2 (flotillin) or non-targeting siRNA (control). Following transfection, some cells were treated with $50 \mu \mathrm{M}$ desipramine for $60 \mathrm{~min}$ followed by USMB treatment, as indicated, which was subsequently followed by incubation with $10 \mu \mathrm{g} / \mathrm{mL} \mathrm{A} 488$-dextran for $30 \mathrm{~min}$, fixation, and imaging by widefield epifluorescence microscopy. (A) Shown are representative fluorescence micrographs showing A488-dextran within cells for each treatment, scale $20 \mu \mathrm{m}$. (B) Shown are the mean \pm SE of total cellular A488-dextran in teach condition, $\mathrm{n}=3$ independent experiments. ${ }^{*} \mathrm{p}<0.05$.

USMB or USMB + desipramine (Fig. 1A,B). These results indicate that flotillins are required for USMB-triggered enhancement of fluid-phase internalization.

The requirement for flotillins in USMB-stimulated enhancement of fluid-phase internalization suggests that USMB may regulate the assembly or dynamics of flotillin structures at the plasma membrane to directly enhance endocytosis, or that flotillins may play a permissive role underlying fluid-phase uptake without being regulated by USMB treatment. To distinguish between these possibilities, we examined the impact of USMB on the dynamics of flotillin2-eGFP in RPE cells, monitored by total internal reflection fluorescence (TIRF) microscopy. Of note, flotillin-1 and -2 exhibited nearly complete overlap (Fig. S2B). As expected ${ }^{10}$, flotillin2-eGFP formed dynamic fluorescent punctate structures at the cell surface in control cells (Fig. 2A). We subjected time-lapse image series to automated detection, tracking and analysis of diffraction-limited flotillin2-eGFP structures ${ }^{48}$. USMB + desipramine treatment did not significantly alter the number (Fig. 2B) or lifetime (Fig. 2C) of flotillin2-eGFP structures but decreased the mean fluorescence intensity of flotillin2-eGFP structures (Fig. 2D). This indicates that $\mathrm{USMB}+$ desipramine treatment regulates flotillin structures at the plasma membrane, which may in turn result in enhancement of flotillin-dependent endocytosis.

Enhanced flotillin-dependent endocytosis will result in decreased abundance of flotillins at the plasma membrane and a concomitant increase of this protein in intracellular compartments ${ }^{24}$. To determine if USMB treatments indeed enhanced flotillin internalization, we next examined the impact of USMB treatment on the plasma membrane localization of endogenous flotillin-1 by immunofluorescence staining and TIRF microscopy, which is highly specific (Fig. S2C) and allows selective illumination of plasma membrane-proximal fluorophores. USMB or USMB + desipramine treatments reduced flotillin-1 fluorescence intensity in the TIRF field $(n=3$, $\mathrm{p}<0.05$, Fig. 2E,F). Treatment with the endocytosis inhibitor dynasore abolished the USMB-elicited reduction in cell surface flotillin-1 (Fig. 2E,F). Flotillin-mediated endocytosis requires dynamin for vesicle scission ${ }^{49}$, and while dynasore was initially reported as a dynamin inhibitor ${ }^{50}$, recent studies have shown that some inhibition of endocytosis by dynasore is dynamin-independent ${ }^{51}$. While these results make conclusions about the specific role of dynamin challenging, the abrogation of the USMB + desipramine-stimulated loss of cell surface flotillin-1 by dynasore treatment suggests that the reduction of cell surface flotillin-1 by USMB + desipramine is likely due to flotillin-1 endocytosis. In addition, we observed no change in cell surface caveolin-1 levels upon identical treatment with USMB + desipramine (Fig. S3), indicating that the effects of this treatment were specific to regulation of flotillin endocytosis. These results collectively indicate that USMB treatment triggers enhanced flotillin-dependent endocytosis, which leads to the internalization of fluid-phase materials.

To determine whether the reduction of flotillin-1 from the cell surface upon USMB treatment contributes to enhanced flotillin-dependent fluid-phase endocytosis, we examined whether USMB stimulation results in overlap of flotillin-1 structures with the fluid-phase marker A488-dextran (Fig. 2G,H). Discernable localized enrichment of A488-dextran within flotillin-1 structures would only occur upon sequestration of this fluid-phase marker within the lumen of internalized vesicles, which is indeed observed in USMB + desipramine treated cells (Fig. 2G). To quantify enrichment of A488-dextran within flotillin structures, we subjected these images to automated detection and analysis of diffraction-limited flotillin-1 puncta ${ }^{48,52}$. USMB + desipramine treatment robustly enhanced the detection of A488-dextran within flotillin structures relative to control samples, or cells only treated with desipramine or USMB alone (Fig. $2 \mathrm{H}$ ). Collectively, these observations indicate that these USMB treatments enhance fluid-phase uptake via regulation of flotillin-dependent endocytosis.

Enhanced flotillin-dependent fluid-phase uptake induced by USMB requires DHHC5. The increase in flotillin-dependent fluid-phase endocytosis by USMB may be related to MEND ${ }^{26}$, and may thus require the palmitoyltransferase DHHC5. To determine if DHHC5 is required for the USMB-stimulated gain in flotillin-dependent fluid-phase endocytosis, we next examined the impact of siRNA gene silencing of 
A
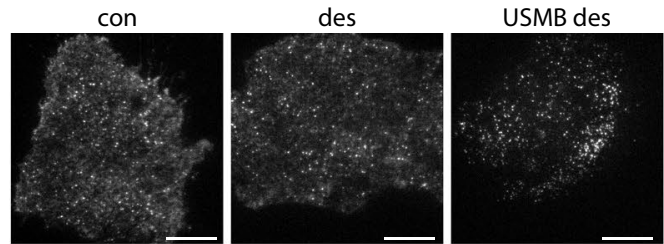

$\mathrm{B}$

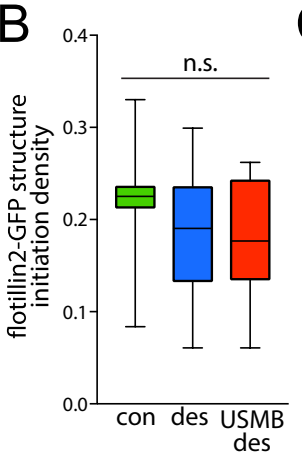

C

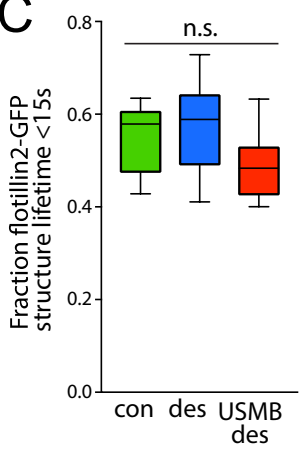

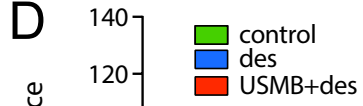

$\left.\begin{array}{ll}D & 140 \\ \Xi & 120\end{array}\right] \quad \begin{aligned} & \text { control } \\ & \text { des } \\ & \text { USMB+des }\end{aligned}$

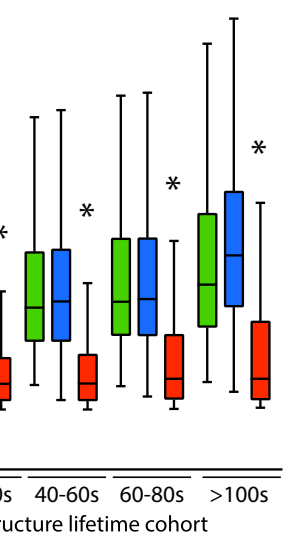

$E$
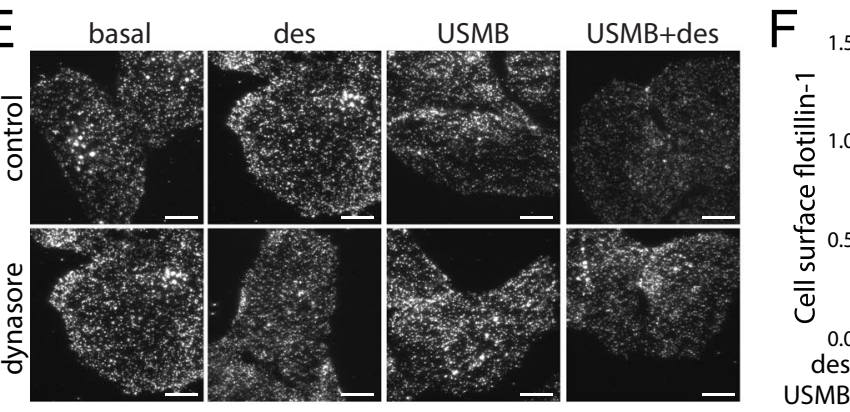

G
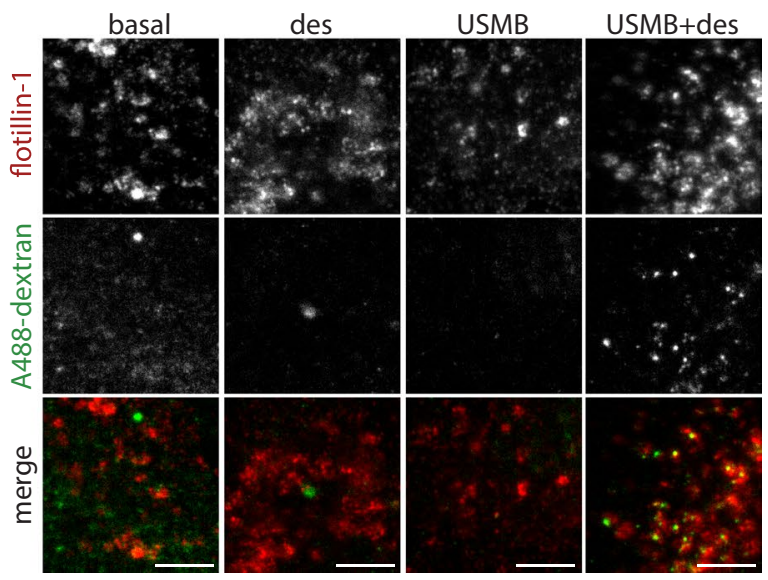

$\mathrm{H}$

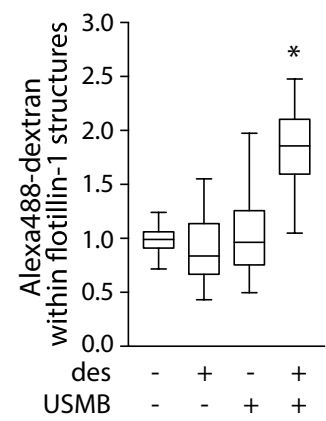

Figure 2. USMB treatment regulates flotillin cell surface levels, dynamics and internalization. (A-D) RPE cells were transfected with cDNA encoding flotillin2-eGFP, then treated with $50 \mu \mathrm{M}$ desipramine (des) for $60 \mathrm{~min}$ followed by USMB treatment, of left untreated (control, con.) as indicated. Cells were then subjected to time-lapse imaging by total internal reflection fluorescence microscopy (TIRF-M), followed by automated detection and analysis of flotillin2-eGFP puncta. Single-frame fluorescence micrographs obtained by TIRF-M are shown in (A). Scale $20 \mu \mathrm{m}$. The initiation density (B), fraction of structures with lifetimes $<15 \mathrm{~s}(\mathbf{C})$ and mean intensity (D) of flotillin2-eGFP structures within indicated lifetime cohorts is shown as the median (line) interquartile range (boxes) and full range (whiskers). ${ }^{*} \mathrm{p}<0.05$, relative to control. The number of cells (k) and flotillin2-eGFP structures (n) in each condition are as follows: control: $\mathrm{k}=8, \mathrm{n}=8626$; des: $\mathrm{k}=12, \mathrm{n}=9757$; USMB + des: $\mathrm{k}=11, \mathrm{n}=8272$. (E,F) RPE cells were treated with $80 \mu \mathrm{M}$ dynasore and/or $50 \mu \mathrm{M}$ desipramine for $60 \mathrm{~min}$, followed by USMB treatment or left untreated, as indicated. Cells were then fixed and subjected to immunofluorescence staining of flotillin-1, and then imaging by TIRF-M. (E) Shown are representative TIRF-M fluorescence micrographs, scale $10 \mu \mathrm{m}$. (F) Flotillin-1 intensity in each cell in TIRF-M images was quantified to determine cell-surface flotillin-1 levels, and these measurements are shown as mean $\pm \operatorname{SE} . n=3$. 
$* \mathrm{p}<0.05(\mathbf{G}, \mathbf{H})$ Cells were treated with $50 \mu \mathrm{M}$ desipramine (des) for $60 \mathrm{~min}$ followed by USMB treatment, or left untreated (control, con.) as indicated. Subsequently, cells were incubated with $10 \mu \mathrm{g} / \mathrm{mL}$ A488-dextran for $30 \mathrm{~min}$, fixed and subjected to staining to detect flotillin-1, and then imaged by spinning disc confocal microscopy. Shown in $(\mathbf{G})$ are representative fluorescence micrographs, scale $5 \mu \mathrm{m}$. (H) Flotillin-1 structures were subjected to automated detection and analysis; shown is the mean cellular A488-dextran intensity detected within flotillin structures in each condition, as median (line), interquartile rand (boxes) and full range (whiskers). The number of cells (k) and flotillin2-eGFP structures (n) in each condition are as follows: control: $\mathrm{k}=49, \mathrm{n}=26774$, des: $\mathrm{k}=49, \mathrm{n}=30701$, USMB: $\mathrm{k}=49, \mathrm{n}=24796$; USMB + des: $\mathrm{k}=47, \mathrm{n}=28751$. *p $<0.05$, relative to control.

DHHC5 (Fig. S4A). While cells subjected to non-targeting (control) siRNA exhibited reduction of $21.5 \pm 0.1 \%$ and $45.0 \pm 0.1 \%$ in cell surface flotillin-1 levels upon USMB or USMB + desipramine treatment, respectively, cells subjected to siRNA silencing of DHHC5 did not (Fig. 3A,B). DHHC5 silencing also prevented the gain in fluid-phase endocytosis elicited by USMB + desipramine treatment that was observed in control siRNA treated cells (Fig. 3C,D). These results indicate that DHHC5 selectively contributes to enhancing flotillin endocytosis and fluid-phase internalization upon USMB treatment. The similar requirement for DHHC5 for the enhanced fluid-phase endocytosis both as triggered by USMB + desipramine and in $\mathrm{MEND}^{26}$ prompted us to examine the requirement for the actin cytoskeleton. Consistent with MEND being unaffected by perturbation of the actin cytoskeleton $^{25}$, latrunculin A (LatA) treatment effectively disrupted filamentous actin, yet was largely without effect on the enhanced fluid-phase endocytosis triggered by USMB + desipramine (Fig. S5).

To determine how USMB may affect the function of DHHC5, we next examined DHHC5 phosphorylation and cell surface localization. DHHC5 localizes at the postsynaptic membrane of neurons through phosphorylation of Y533 in a manner regulated by Fyn ${ }^{34}$. To test the possible regulation of DHHC5 by Fyn and/or phosphorylation upon USMB treatment, we first examined the phosphorylation of DHHC5 using phos-tag gel electrophoresis, a method that exaggerates the apparent molecular weight differences elicited by protein phosphorylation $^{53}$. We observed that USMB and USMB + desipramine elicited a reduction in the apparent molecular weight of DHHC5 observed by phos-tag gel (Fig. 4A, left lanes), suggesting that USMB induces DHHC5 dephosphorylation. Interestingly, silencing of Fyn (Fig. S6A) impaired the apparent shift in DHHC5 molecular weight induced by USMB and USMB + desipramine (Fig. 4A, right lanes). These results indicate that USMB treatment leads to a reduction of DHHC5 phosphorylation, and that this phenomenon is controlled by Fyn.

We next examined how USMB treatment may alter the abundance of endogenous DHHC5 at the plasma membrane using TIRF microscopy. USMB + desipramine treatment increased DHHC5 intensity detected in the TIRF field, and this gain in DHHC5 levels in the TIRF field upon USMB + desipramine treatment was ablated in cells subjected to Fyn silencing (Fig. 4B,C). This indicates that Fyn controls both the regulation of DHHC5 phosphorylation as well as the gain in cell surface abundance of DHHC5 triggered by USMB treatments.

Phosphorylation of DHHC5 at Y533 controls its cell surface abundance ${ }^{34}$. We next examined the effect of expression of a phosphorylation deficient Y533A mutant of DHHC5 on cell surface flotillin-1 levels following USMB treatments. RPE cells were transfected with wild-type eGFP-DHHC5(WT) or eGFP-DHHC5(Y533A) constructs followed by treatment with USMB, USMB + desipramine or desipramine alone. As expected, treatment of cells expressing eGFP-DHHC5(WT) with USMB or USMB + desipramine resulted in decreased levels of flotillin-1 at the cell surface compared to cells not treated with either USMB or desipramine (basal) (Fig. 5A,B, black bars). In contrast, in cells transfected with eGFP-DHHC5(Y533A), treatment with USMB or USMB + desipramine had no significant effect on the cell surface flotillin-1 levels compared to cells not treated with USMB (Fig. 5A,B, grey bars). Collectively these results show a functional requirement for regulation of DHHC5 in USMB-stimulated enhancement of flotillin-dependent fluid-phase endocytosis.

Enhanced flotillin-dependent fluid-phase uptake induced by USMB requires Fyn. We observed that Fyn was required for the regulation of DHHC5 phosphorylation (Fig. 4A) and for the gain in cell surface DHHC5 (Fig. 4C) that occur upon USMB treatment. To determine whether USMB-mediated reduction of cell surface flotillin-1 requires Fyn, we used siRNA gene silencing of Fyn (Fig. S6A). USMB or USMB + desipramine treatment elicited a reduction of flotillin-1 from the cell surface in cells transfected with non-targeting (control) siRNA (Fig. 6A,B, black bars). In contrast, cells treated with siRNA to silence Fyn did not exhibit a change in cell surface flotillin-1 levels upon USMB or USMB + desipramine treatments (Fig. 6A,B, grey bars), suggesting that Fyn is required for USMB-induced flotillin endocytosis. In addition, cells subjected to siRNA to silence Fyn did not exhibit an increase in fluid-phase endocytosis following USMB or USMB + desipramine treatments, measured by A488-dextran uptake, as was observed in cells subjected to non-targeting (control) siRNA (Fig. 6C,D). These results indicate that Fyn is required for the enhanced flotillin-dependent fluid-phase endocytosis elicited by USMB treatment.

Enhanced flotillin-dependent uptake induced by USMB enhances cytotoxic drug uptake and action. USMB treatments thus elicit a robust increase in flotillin-dependent fluid-phase endocytosis that also requires DHHC5 and Fyn. To determine how this novel signaling pathway may contribute to enhanced uptake of chemotherapeutic drugs, and whether the increase in drug uptake can potentiate the action of chemotherapeutic drugs on cell viability, we first examined this phenomenon in RPE cells. Following treatment with USMB or $\mathrm{USMB}+$ desipramine, cells were incubated in a solution containing $30 \mu \mathrm{M}$ cisplatin (CDDP) for $2 \mathrm{~h}$, followed by drug washout; cell viability was assessed $24 \mathrm{~h}$ later. This experimental design allowed for the enhanced fluid-phase endocytosis elicited by USMB treatments to cause an increase the uptake of cisplatin (for a $2 \mathrm{~h}$ period) through 
A

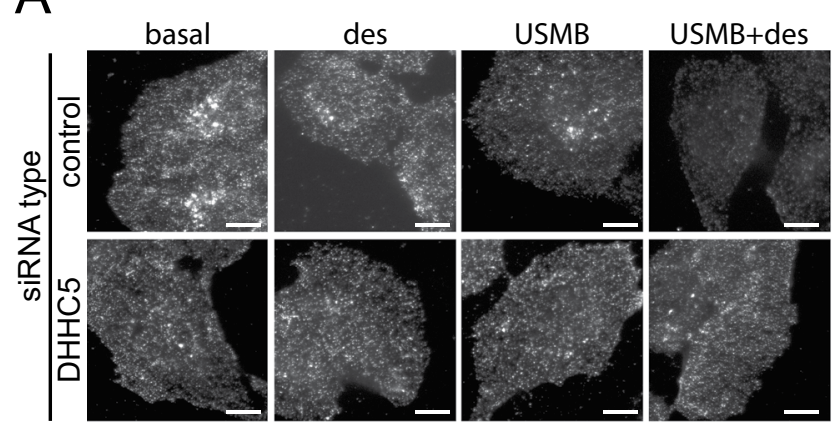

C

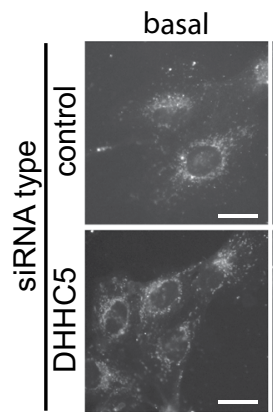

des

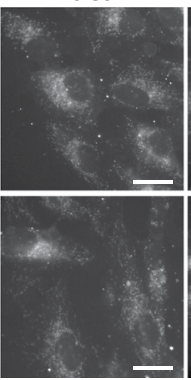

USMB

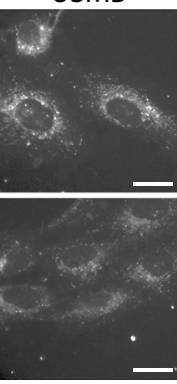

USMB+des

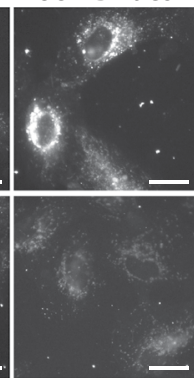

control siRNA DHHC5 SiRNA
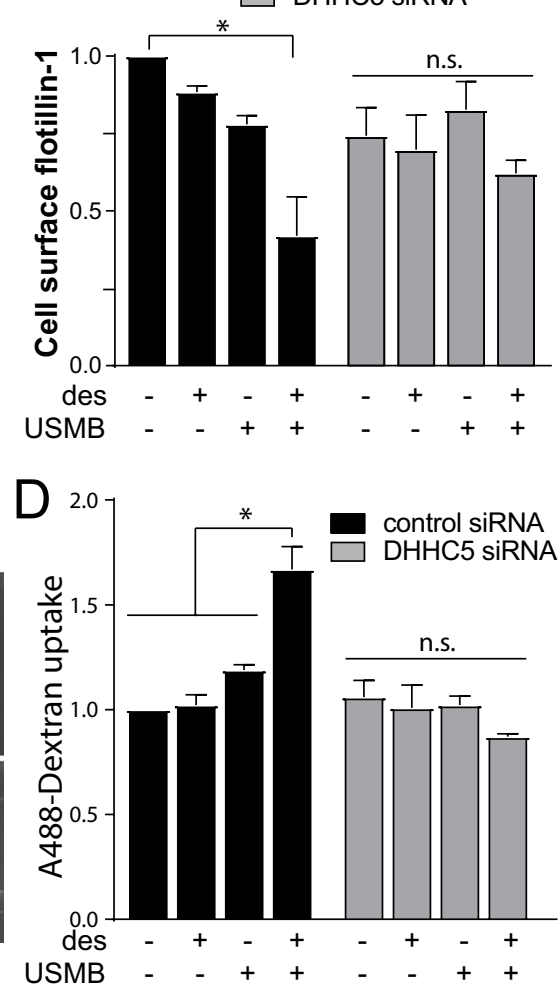

Figure 3. DHHC5 is required for USMB-triggered flotillin and fluid-phase internalization. RPE cells were transfected with siRNA targeting DHHC5 or non-targeting siRNA (control). Following transfection, cells were treated with $50 \mu \mathrm{M}$ desipramine for $60 \mathrm{~min}$ followed by USMB treatment. (A,B) Following treatments and a $30 \mathrm{~min}$ incubation, cells were fixed and subjected to immunofluorescence staining of flotillin-1, and then imaged by TIRF-M. Shown in (A) are representative TIRF-M fluorescence micrographs, scale $10 \mu \mathrm{m}$. Flotillin-1 intensity in each cell in TIRF-M images was quantified to determine cell-surface flotillin-1 levels, and these measurements are shown in $(\mathbf{B})$ as mean \pm SE. $n=3 .{ }^{*} \mathrm{p}<0.05$. (C,D) Following treatments, cells were incubated with $10 \mu \mathrm{g} / \mathrm{mL}$ A488-dextran for $30 \mathrm{~min}$, fixed, and imaged by widefield epifluorescence microscopy. Shown in (C) are representative fluorescence micrographs showing A488-dextran within cells for each treatment, scale $20 \mu \mathrm{m}$. Shown in (D) are the mean \pm SE of total cellular A488-dextran in each condition, $\mathrm{n}=3$. $* \mathrm{p}<0.05$. Experiments shown in $(\mathbf{A}, \mathbf{B})$ were performed separately from those shown in $(\mathbf{C}, \mathbf{D})$.

enhancement of fluid-phase endocytosis by USMB treatment, followed by assessment of the outcome of this enhanced drug uptake on viability at a later point ( $24 \mathrm{~h}$ later). Importantly, cells treated with USMB + desipramine in combination with cisplatin exhibited a significant reduction in cell viability compared to cells treated with either USMB, USMB + desipramine, USMB + cisplatin or cisplatin alone (Fig. S7A).

To determine if USMB + desipramine treatment prior to chemotherapy drug treatment resulted in enhanced uptake and retention of the drug at the time of assessment of cell viability, we repeated similar experimental treatments with USMB and USMB + desipramine, followed by incubation for $2 \mathrm{~h}$ in doxorubicin, a cancer chemotherapeutic drug that is intrinsically fluorescent. Cells treated with USMB + desipramine prior to $2 \mathrm{~h}$ incubation with doxorubicin exhibited a significantly higher level of cellular doxorubicin assessed $24 \mathrm{~h}$ subsequent to drug incubation (Fig. S7B,C). Doxorubicin internalization and/or retention may be regulated by caveolae ${ }^{54,55}$, but as we did not observe changes in cell surface caveolin-1 upon USMB + desipramine treatment, and the enhanced fluid-phase internalization triggered by USMB + desipramine was entirely flotillin-1-dependent, the enhanced intracellular doxorubicin observed in this condition is likely not due to enhanced caveolae-mediated internalization. Together, these results indicate that USMB + desipramine treatment results in enhanced fluid-phase internalization, which contributes to enhanced uptake and action of cytotoxic cancer drugs on cell viability.

To examine how stimulation of fluid-phase endocytosis by USMB + desipramine may contribute to the enhanced uptake and consequently the potentiated action of cisplatin in cancer cells, we next examined the impact of this treatment in MDA-MB-231 cells, a model of triple-negative breast cancer ${ }^{53}$. We used CRISPR/ Cas9 genome editing to generate an MDA-MB-231 cell line deficient in flotillin-1, which also exhibited substantial reduction in expression of flotillin-2, as expected ${ }^{56}$ (MDA-MB-231-flot1-KO, Fig. S7D). While wild-type MDA-MB-231 cells exhibited a robust increase in fluid-phase internalization upon treatment with USMB + desipramine, MDA-MB-231-flot1-KO cells exhibited no discernable change in fluid-phase internalization upon this treatment (Fig. 7A,B). 


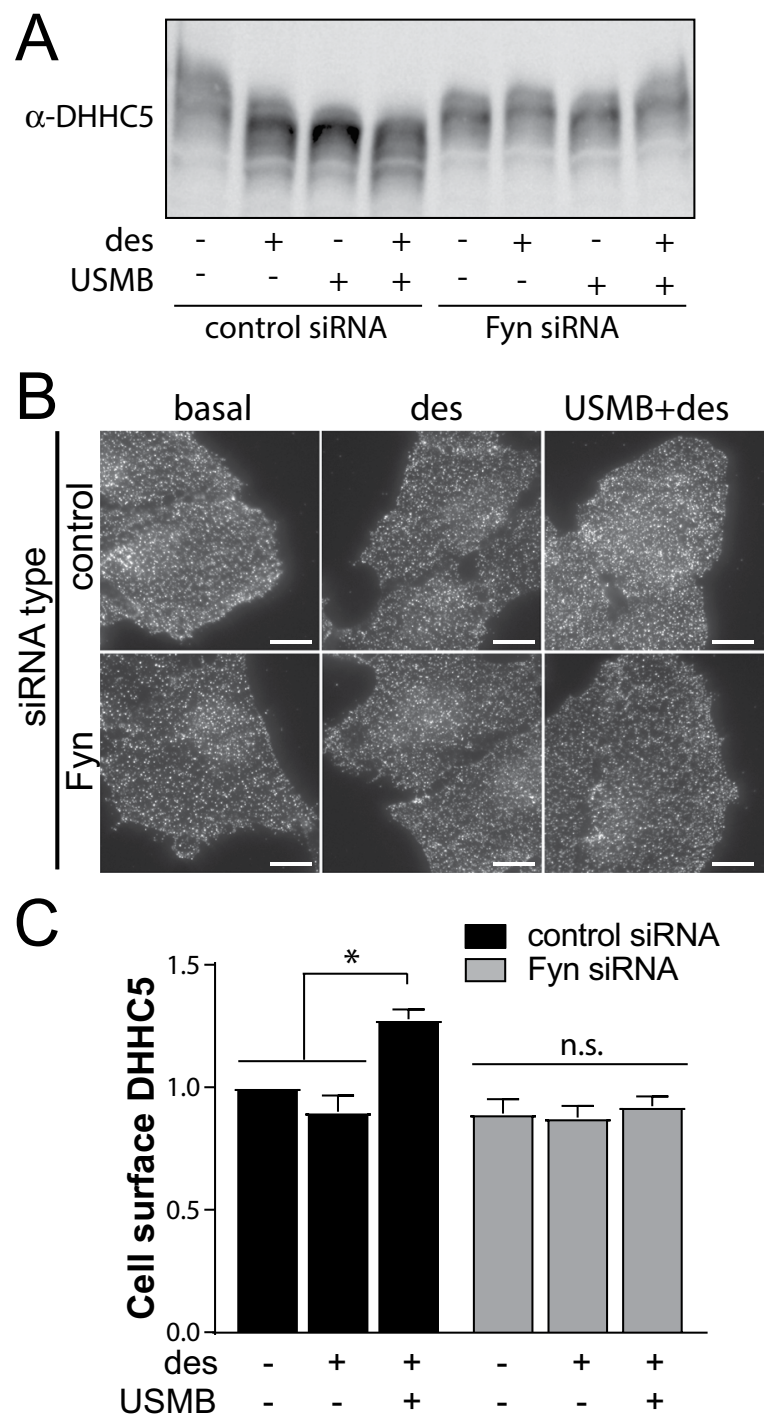

Figure 4. USMB treatment regulates DHHC5. RPE cells were transfected with siRNA targeting Fyn or nontargeting siRNA (control). Following transfection, some cells were treated with $50 \mu \mathrm{M}$ desipramine for $60 \mathrm{~min}$ followed by USMB treatment, as indicated. (A) Following treatments, cells were lysed and whole-cell lysates were resolved by phos-tag SDS-PAGE and western blotting to detect DHHC5. Shown is a representative immunoblot showing DHHC5 staining. Full blot images are available in Fig. S8. (B,C) Following treatments and a subsequent $30 \mathrm{~min}$ incubation, cells were then fixed and subjected to immunofluorescence staining of DHHC5, and then imaging by TIRF-M. Shown in (B) are representative TIRF-M fluorescence micrographs, scale $10 \mu \mathrm{m}$. DHHC5 intensity in each cell in TIRF-M images was quantified to determine cell surface flotillin-1 levels, and these measurements are shown in $(\mathbf{C})$ as mean \pm SE. $n=3 .{ }^{*} \mathrm{p}<0.05$.

We next examined the impact of this treatment on cisplatin-mediated loss of cell viability. To do so, we again treated cells for $2 \mathrm{~h}$ with $30 \mu \mathrm{M}$ cisplatin immediately following the USMB treatment, followed by drug washout and measurement of cell viability $24 \mathrm{~h}$ later. Wild-type MDA-MB-231 cells treated with cisplatin only (no USMB + desipramine) exhibited a modest decrease in viability compared to control cells (not treated with either USMB + desipramine or cisplatin) (Fig. 7C). Importantly, wild-type MDA-MB-231 cells treated with $\mathrm{USMB}+$ desipramine prior to cisplatin treatment exhibited a robust increase in cell death compared to either cells treated only with USMB + desipramine or cisplatin alone. Strikingly, MDA-MB-231-flot1-KO cells exhibited no change in viability upon treatment with either cisplatin, USMB + desipramine, or the two treatments combined (Fig. 7C).

Cisplatin leads to a reduction in cell viability by eliciting DNA damage in the form of double-strand breaks, which first triggers repair mechanisms that can be detected by the presence of $\gamma \mathrm{H} 2 \mathrm{AX}$ nuclear puncta ${ }^{57}$. As expected, in wild-type MDA-MB-231 cells, cisplatin treatment alone elicited an increase in $\gamma \mathrm{H} 2 \mathrm{AX}$ levels, which was significantly enhanced by prior treatment with USMB + desipramine (Fig. 7D,E). Importantly, and consistent with the outcome of cell viability assays, MBD-MB-231-flot-KO cells exhibited no discernable enhancement of $\gamma \mathrm{H} 2 \mathrm{AX}$ levels by cisplatin upon prior treatment with USMB + desipramine (Fig. 7D,E). Collectively, these 
A

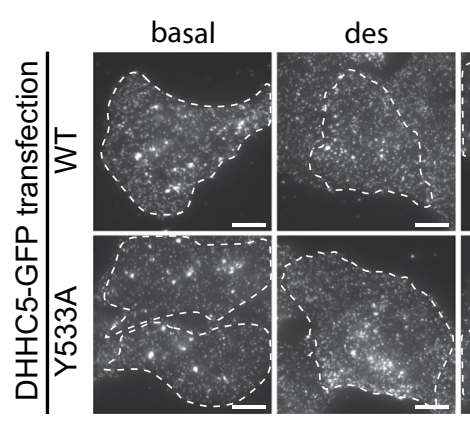

USMB

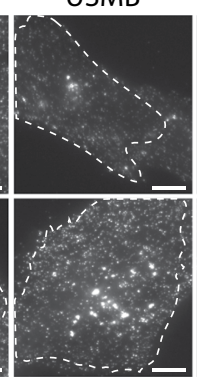

USMB+des

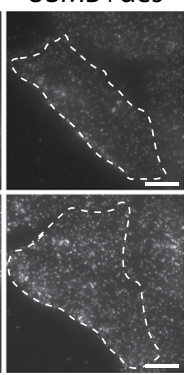

B

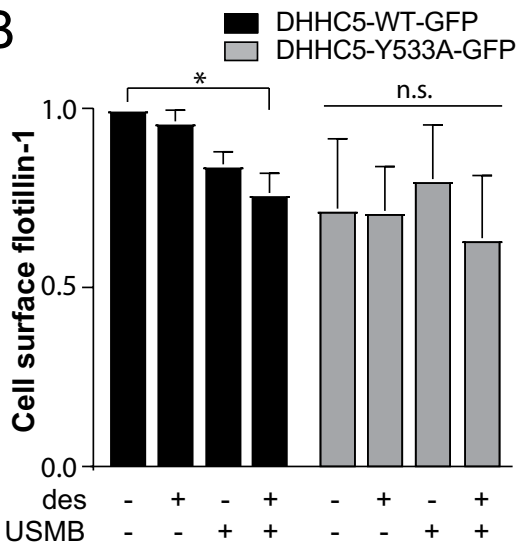

Figure 5. Expression of a phosphorylation-defective mutant of DHHC5 impairs flotillin internalization elicited by USMB treatment. RPE cells were transfected with cDNA encoding either wild-type (WT) or Y533A mutant DHHC5, fused to eGFP. Following transfection, cells were treated with $50 \mu \mathrm{M}$ desipramine for $60 \mathrm{~min}$ followed by USMB treatment. Following a subsequent $30 \mathrm{~min}$ incubation, cells were then fixed and subjected to immunofluorescence staining of flotillin-1, and then imaging by TIRF-M. (A) Shown are representative TIRF-M fluorescence micrographs, scale $10 \mu \mathrm{m}$. Transfected cells are outlined, and GFP-channel images are shown in Fig. S4B. (B) Flotillin-1 intensity in each cell in TIRF-M images was quantified to determine cellsurface flotillin-1 levels, and these measurements are shown as mean $\pm \mathrm{SE} . \mathrm{n}=3$. ${ }^{*} \mathrm{p}<0.05$.

experiments indicate that the enhanced flotillin-dependent fluid-phase internalization triggered by USMB treatments elicits enhanced cisplatin uptake, as well as increased DNA damage and cell death by cisplatin.

\section{Discussion}

We identified that enhancement of flotillin-dependent endocytosis may be a useful strategy for enhancing drug delivery in cancer treatment. This represents one of the first demonstrations that interventions to enhance fluid-phase endocytosis can mediate enhanced drug delivery into cells. The ability to clinically focus ultrasound on small volumes may thus allow targeted drug delivery localized to a specific part of a tissue such as a tumor. Moreover, we identified that flotillin-dependent endocytosis triggered by USMB requires the palmitoyltransferase DHHC5 and the Src-family kinase Fyn, and we further identified that USMB induces regulation of DHHC5 that requires Fyn and may require modulation of DHHC5 phosphorylation. This led us to propose a signaling pathway that links USMB treatment to stimulation of flotillin-dependent endocytosis that initiates with Fyn engagement and control of the palmitoyltransferase DHHC5 (Fig. 8). Finally, we uncovered that the USMB-stimulated enhancement of flotillin-dependent fluid-phase endocytosis may be significant for enhancing the delivery of drugs such as cisplatin to cancer cells.

The role of DHHC5 in USMB-triggered enhancement of fluid-phase endocytosis suggests that this endocytic mechanism may be similar to $\mathrm{MEND}^{26}$. MEND is triggered by the opening of mitochondrial permeability transition pore channels, which leads to the enhancement of DHHC5-dependent endocytosis of ordered membrane domains ${ }^{26}$. The requirement for DHHC5 in the internalization of ordered membrane domains is consistent with our observation that USMB triggers flotillin internalization in a DHHC5-depenedent manner ${ }^{47}$. Like observed for MEND ${ }^{25}$, we also found that the enhanced fluid-phase endocytosis triggered by USMB is also largely insensitive to actin perturbation (Fig. S5). Our work is novel in that we identified the involvement of flotillin in DHHC5-dependent endocytosis, and found that the stimulation of this pathway can lead to enhanced drug delivery into cells. MEND triggers internalization of $\sim 50-70 \%$ of cell surface area ${ }^{26}$, which is at least approximately in line with a $20-50 \%$ enhancement in fluid-phase endocytosis that we observe here.

Our results indicate that USMB treatments may trigger a MEND-like phenomenon, leading to DHHC5-dependent enhancement of flotillin endocytosis. Flotillins themselves are palmitoylated ${ }^{15,58,59}$, and DHHC5 palmitoylates flotillin- $2^{17}$, suggesting that direct and specific palmitoylation of flotillins by DHHC5 could enhance fluid-phase endocytosis. However, as selective perturbation of flotillin palmitoylation by site-directed mutagenesis of cysteine residues known to be palmitoylated leads to retention of flotillin-1 in the $\mathrm{ER}^{56}$ or loss of membrane association of flotillin- $2^{15}$, these strategies are unlikely to reveal specific roles for dynamic palmitoylation in response to treatments such as USMB. DHHC5 has several identified substrates and as such our study does not resolve which of the many DHHC5 substrate(s) may be subject to palmitoylation upon USMB and desipramine treatment to trigger enhanced endocytosis in this condition, as was suggested to occur in $\mathrm{MEND}^{26}$. Nonetheless, whether USMB treatments elicit broad palmitoylation or palmitoylation of only a few key protein(s) to trigger enhancement of fluid-phase endocytosis, our results strongly indicate that this is flotillin-dependent (Fig. 1), and results in enhanced formation of flotillin-positive carriers derived from the plasma membrane (Fig. 2).

How might USMB treatments control flotillin internalization to increase fluid-phase internalization and thus enhance drug delivery? Tracking of flotillin2-eGFP structures in living cells (Fig. 2A-D) revealed that USMB stimulation led to a reduction of flotillin2-eGFP intensity in these flotillin structures (Fig. 2D), consistent with 
A
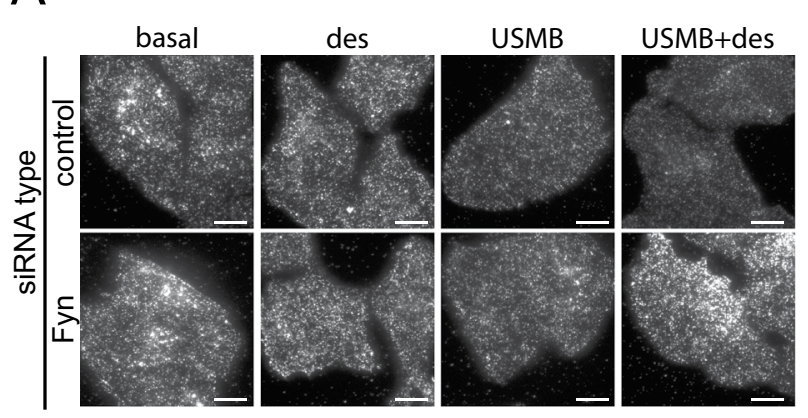

C
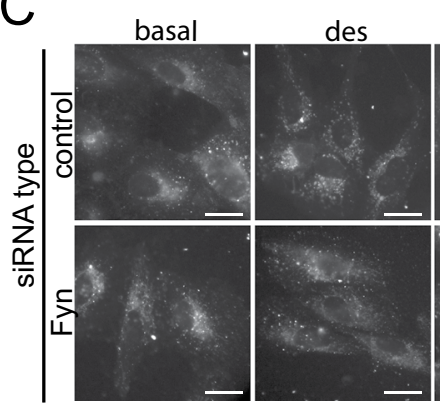

USMB

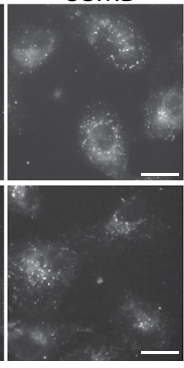

USMB+des

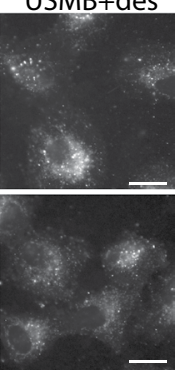

B
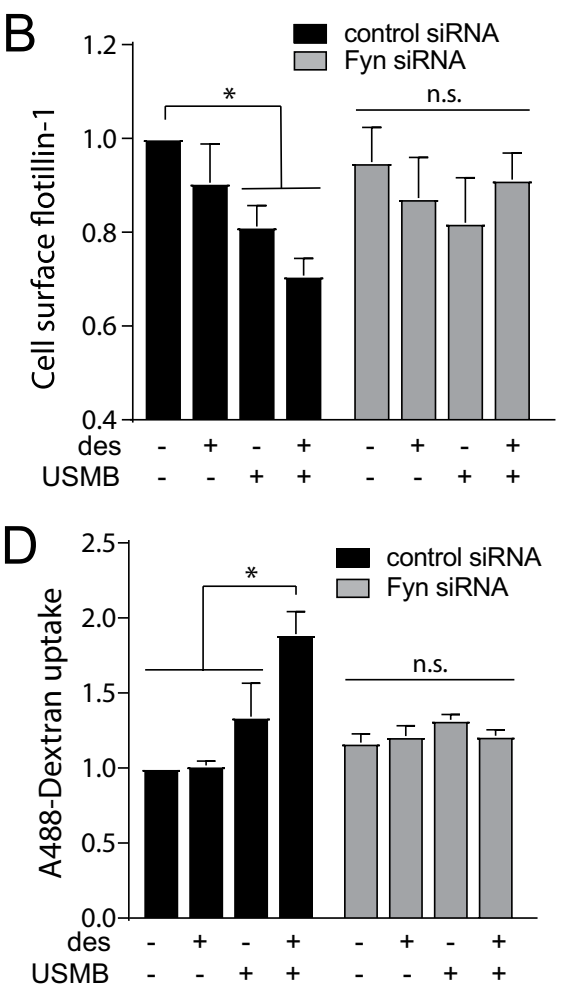

Figure 6. Fyn is required for USMB-triggered flotillin and fluid-phase internalization. RPE cells were transfected with siRNA targeting Fyn or non-targeting siRNA (control). Following transfection, some cells were treated with $50 \mu \mathrm{M}$ desipramine for $60 \mathrm{~min}$ followed by USMB treatment, as indicated. (A,B) Following treatments and a $30 \mathrm{~min}$ incubation, cells were fixed and subjected to immunofluorescence staining of flotillin-1, and then imaged by TIRF-M. Shown in (A) are representative TIRF-M fluorescence micrographs, scale $10 \mu \mathrm{m}$. Flotillin-1 intensity in each cell in TIRF-M images was quantified to determine cell surface flotillin-1 levels, and these measurements are shown in $(\mathbf{B})$ as mean \pm SE. $n=3$ independent experiments. ${ }^{*} \mathrm{p}<0.05(\mathbf{C}, \mathbf{D})$ Following treatments, cells were incubated with $10 \mu \mathrm{g} / \mathrm{mL}$ A488-dextran for $30 \mathrm{~min}$, fixed, and imaged by widefield epifluorescence microscopy. Shown in (C) are representative fluorescence micrographs showing A488-dextran in cells of each treatment, scale $10 \mu \mathrm{m}$. Shown in (D) are the mean \pm SE of total cellular A488-dextran in each condition, $n=3$ independent experiments. ${ }^{*} \mathrm{p}<0.05$. In panel (D) comparison of the control siRNA control (no USMB or des) and control siRNA USMB (no des.) condition resulted $\mathrm{p}=0.052$ (not significantly different by our threshold). Experiments shown in (A,B) were performed separately from those shown in $(\mathbf{C}, \mathbf{D})$.

USMB stimulation leading to a reduction of flotillin at the cell surface (Fig. 2E). That USMB treatment decreases the intensity of flotillin2-eGFP in flotillin structures suggests that this treatment may either restrict flotillin oligomerization or may selectively enhance endocytic turnover of larger flotillin structures. This DHHC5-dependent regulation of flotillin-dependent endocytosis adds to the known regulation of flotillin-dependent endocytosis by phosphorylation ${ }^{24}$. A detailed understanding of the molecular mechanism by which flotillin contributes to endocytosis is currently lacking, which limits more detailed understanding of how USMB treatments and other cues may modulate this process. Future studies thus aimed at elucidating this molecular mechanism will be of great interest.

We identify Fyn and DHHC5 as upstream regulators of flotillin-dependent endocytosis, as perturbations of either DHHC5 (Fig. 3) or Fyn (Fig. 6) impair the USMB-stimulated reduction of cell surface flotillin-1 and enhancement of fluid-phase endocytosis (Figs. 4 and 6). Fyn may function upstream of DHHC5 (Fig. 8) based on the observation that Fyn silencing prevented the USMB-stimulated changes in DHHC5 phosphorylation (Fig. 4A) and cell surface DHHC5 levels (Fig. 4C). While we also observed that Fyn contributes to the regulation of DHHC5 phosphorylation (Fig. 4A), this appears to be a distinct mechanism from what was proposed in neurons ${ }^{34}$, as in the latter, Fyn was proposed to directly phosphorylate DHHC5 upon binding to PSD95, leading to impairment of DHHC5 endocytosis. In contrast, our results suggest that Fyn is part of a signaling mechanism engaged upon USMB and desipramine treatment that leads to DHHC5 dephosphorylation. Moreover, DHHC5 dephosphorylation occurs upon treatment with either desipramine or USMB alone (Fig. 4A), conditions which are largely unable to trigger a robust enhancement of fluid-phase internalization on their own (e.g. Fig. 1). As such, DHHC5 dephosphorylation, as triggered by either USMB or desipramine treatments, may not be sufficient to elicit an increase in fluid-phase internalization, and additional mechanisms of regulation of DHHC5 may contribute to this phenomenon. Consistent with this, both wild-type and Y533A mutant of DHHC5 are detected in the TIRF field in both basal and USMB treatment conditions (Fig. S4), which argues 

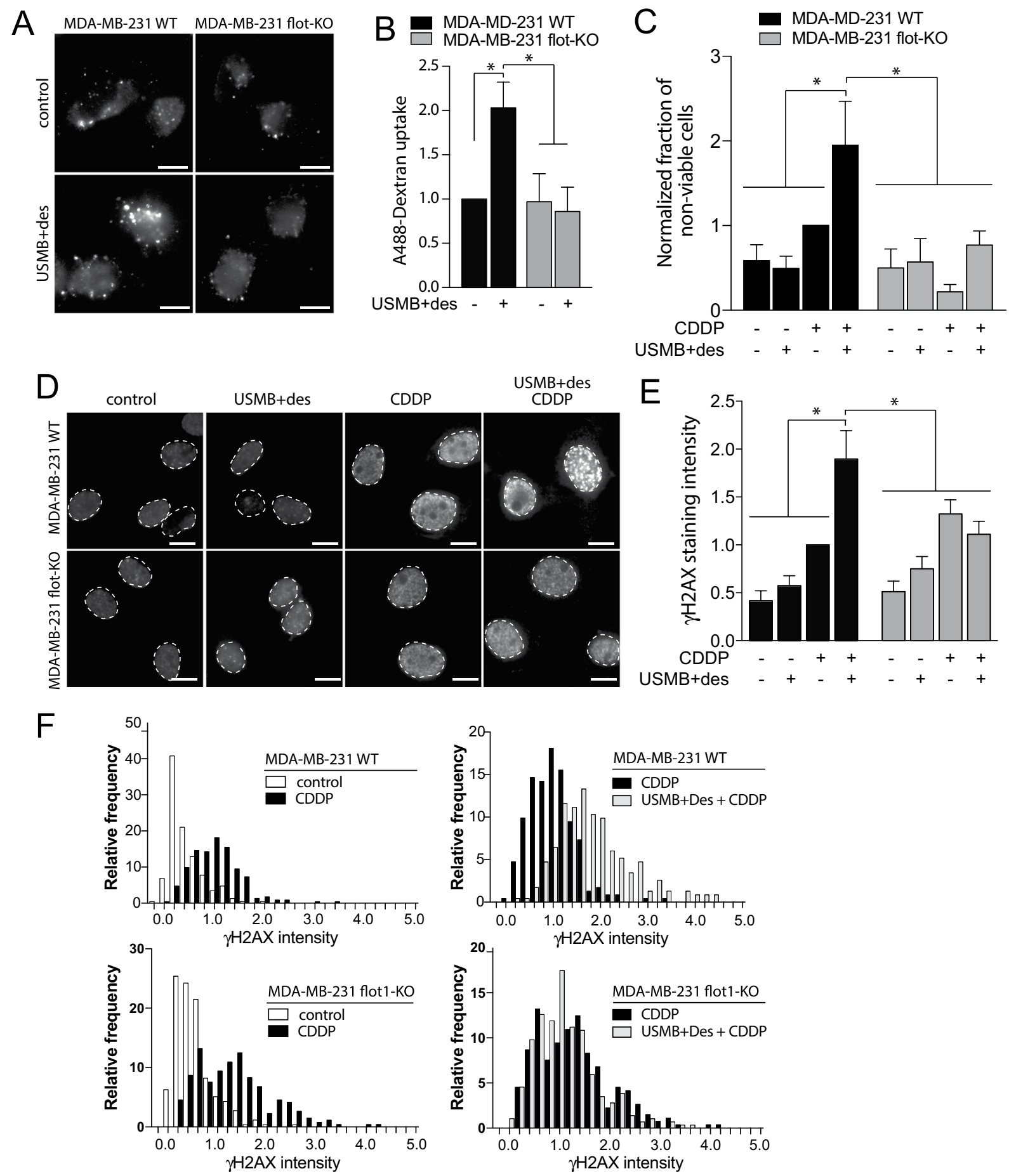

Figure 7. USMB treatments enhance cisplatin-mediated DNA damage and death in breast cancer cells in a flotillin-1 dependent manner. MDA-MB-231 wild-type (WT) or flotillin knockout (flot-KO) cells were treated with $50 \mu \mathrm{M}$ desipramine for $60 \mathrm{~min}$ followed by USMB treatment, as indicated. (A,B) Following treatments, cells were incubated with $10 \mu \mathrm{g} / \mathrm{mL}$ A488-dextran for $30 \mathrm{~min}$, fixed, and imaged by widefield epifluorescence microscopy. Shown in (A) are representative fluorescence micrographs showing A488-dextran in cells of each treatment, scale $20 \mu \mathrm{m}$. Shown in (B) are the mean \pm SE of total cellular A488-dextran in each condition, $\mathrm{n}=3$ independent experiments. $* \mathrm{p}<0.05$. (C-F) Following USMB and other treatments, cells were incubated with $30 \mu \mathrm{M}$ cisplatin (CDDP) for $2 \mathrm{~h}$ (as shown), followed by washing and incubation in growth media (no drugs) (C) $24 \mathrm{~h}$ after USMB and/or cisplatin exposure, cell viability was assessed and shown are the mean $\pm S E$ of the fraction of non-viable cells, normalized to that of MDA-MB-231 WT cells treated with cisplatin only. $\mathrm{n}=3$ independent experiments. $* \mathrm{p}<0.05$. (D-F) $24 \mathrm{~h}$ after USMB and/or cisplatin exposure, cells were fixed and stained to detect $\gamma \mathrm{H} 2 \mathrm{AX}$. Shown in (D) are representative fluorescence micrographs for each condition, scale $10 \mu \mathrm{m}$. Shown in (E) is the mean $\pm \mathrm{SE}$ of $\gamma \mathrm{H} 2 \mathrm{AX}$ intensity in each independent experiment. $\mathrm{n}=3$ independent experiments. ${ }^{*} \mathrm{p}<0.05$. Also shown in (F) are frequency distributions of $\gamma \mathrm{H} 2 \mathrm{AX}$ intensity measurements in individual cells. 


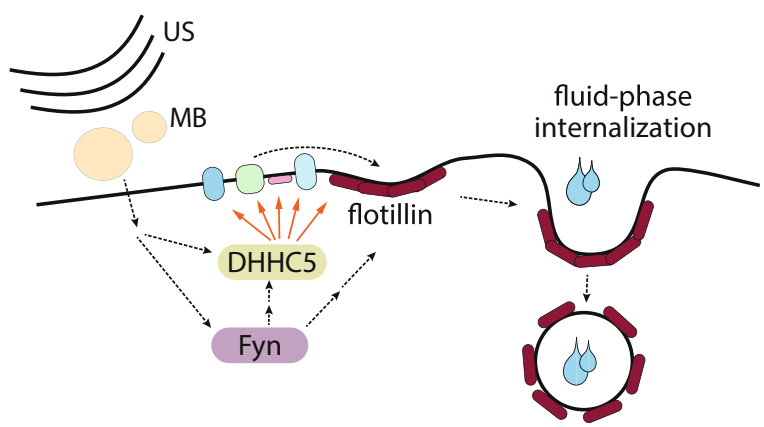

Figure 8. Model of USMB-triggered enhancement of flotillin-dependent fluid-phase internalization. Shown is a diagram depicting ultrasound (US) and microbubble (MB) stimulation, which leads to activation of a signaling pathway involving Fyn and DHHC5, which in turn triggers an increase in flotillin-dependent internalization. This phenomenon elicits an enhancement of fluid-phase internalization, which leads to increased cellular uptake of hydrophilic drugs. In the case of genotoxic drugs such as cisplatin, USMB treatments lead to increased DNA damage and loss of cell viability.

against robust alterations of DHHC5 membrane traffic dependent on phosphorylation of the Y533 residue. As such, the requirement for an intact Y533 residue on DHHC5 to support flotillin internalization upon USMB and desipramine stimulation (Fig. 5) may reflect more complex regulation of DHHC5, such cycling between phosphorylation-dephosphorylation and/or appropriate subcellular localization of DHHC 5 that is disrupted by the DHHC5 Y533A mutation. Our results nonetheless suggest that Fyn is required for USMB-induced dephosphorylation of DHHC5 in non-neuronal cells as well as an increase in cell surface DHHC5 (Fig. 4), suggesting additional mechanism by which Fyn controls DHHC5 distinct from direct phosphorylation.

Perturbation of flotillin, DHHC5 or Fyn selectively impairs fluid-phase internalization upon USMB treatments, but not in resting cells (Figs. 1, 3 and 6). Previous studies indeed found that flotillin perturbation impairs fluid-phase endocytosis ${ }^{10}$, but the contribution of flotillins relative to other forms of clathrin-independent endocytosis varies by cell and condition context ${ }^{9}$. These results suggest that other endocytic mechanisms are largely responsible for constitutive fluid-phase endocytosis in resting cells. Importantly, flotillin-dependent endocytosis can thus be selectively triggered to boost fluid-phase endocytosis in a selective manner in response to USMB stimulation, indicating that this may be an effective strategy for the selective and targeted delivery of drugs into cancer cells.

USMB has been increasingly studied as an attractive and exciting strategy for targeted drug delivery in cancer therapy. Indeed, USMB treatment can lead to enhanced drug molecule uptake and/or action in vivo ${ }^{60-62}$ which may be linked to additional effects of USMB such as enhancement of vascular permeability near tumors ${ }^{63}$. At the cellular level, USMB treatment elicits the formation of transient pores, a phenomenon termed sonoporation ${ }^{43}$. While sonoporation has been proposed as a strategy for the delivery of drugs from the extracellular milieu by simple diffusion, these pores are transient and typically re-seal within $<1 \mathrm{~min}^{44}$. As such, these transient pores may only have limited direct contribution to cellular drug uptake. Most experimental strategies that assess the ability of USMB to enhance drug uptake involve pre-treatment of cells with the drug in question, and then ongoing incubation in the drug solution following USMB stimulation. Hence, these strategies do not differentiate whether the route of drug entry is through the transient pores that form and re-seal during or immediately after USMB ( $<1 \mathrm{~min})$, or through the sustained enhancement of fluid-phase endocytosis as we ${ }^{47}$ and others $^{43-46}$ have observed. Here, we added cisplatin starting at 5 min following USMB stimulation, thus allowing us to exclude direct entry through USMB-induced pores as a mechanism for enhanced cellular uptake of cisplatin and enhanced cisplatin-mediated cell killing in USMB-treated cells. That enhanced endocytic uptake via flotillin-dependent endocytosis and not direct entry via USMB-induced transient pores mediates the enhanced cisplatin uptake and action upon USMB treatment is further supported by: (i) the punctate distribution of fluid phase makers upon entry into cells and (ii) that the effect of USMB to enhance cell killing by cisplatin is completely abrogated in flotillin-1 knockout cells (Fig. 7C).

Our work indicates that USMB, in particular in combination with systemic administration of desipramine, may be an effective combination to selectively enhance drug uptake in cancer cells, but that additional studies of tissue penetration and pharmacokinetics of desipramine or microbubbles may be required. This may a particularly effective strategy to achieve targeted drug delivery, as USMB is currently used in the clinic as a diagnostic tool and desipramine is also a clinically-approved agent. Notably, desipramine is without effect on fluid-phase internalization in the absence of USMB, indicating that systemic administration of desipramine and microbubbles, followed by targeted, focused ultrasound can be used for targeted enhanced drug delivery (e.g. to tumors). The identification of the molecular target of desipramine in future studies may extend this proof-of-concept work to additional clinical applications.

The augmentation of cisplatin-induced death in cells treated with USMB and desipramine suggests that fluid-phase internalization via flotillin-mediated endocytosis may be responsible for the enhanced cisplatin delivery into cells, which in turn elicits higher level of DNA damage (Fig. 7D,E), leading to reduced cell viability. This is consistent with a significantly more potent effect of the combination of USMB and desipramine to enhance the effect of cisplatin on cell viability relative to USMB alone (Fig. S7A), the latter which also exhibited only a modest 
effect on enhancing fluid-phase uptake, substantially less than the effect of USMB and desipramine combined (e.g. Fig. 1). This in turn implies not only that cisplatin is taken up into intracellular vesicles, but also that this treatment results in uptake that has slow recycling of internalized vesicles leading to sustained exposure of cells to drugs within the fluid-phase lumen of these vesicles. Consistent with this, we observed that doxorubicin was retained in USMB-treated cells to a higher extent than in control cells (Fig. S7B,C). Moreover, the increased ability of cisplatin to trigger cell death and DNA damage in USMB-treated cells following flotillin-dependent endocytosis suggests that a significant amount of cisplatin must translocate from the lumen of internalized vesicles to access the nucleus (Fig. 7D,E). The mechanism by which this may occur is unclear and could be due to simple diffusion from the lumen of internalized vesicles retained within the cell or other mechanisms ${ }^{4,5}$.

In conclusion, flotillin-dependent endocytosis is an endocytic pathway that can be modulated by therapeutically-compatible manipulations such as USMB treatments in order to enhance fluid-phase uptake of cytotoxic drugs, leading to enhanced action of these drugs on cancer cells. This highlights the potential usefulness of USMB or other therapies that control fluid-phase endocytosis in developing more effective cancer treatments, by allowing targeted drug delivery and uptake into tumors or other diseased tissues.

\section{Methods}

Materials. Antibodies to detect flotillin-1 $\gamma, \mathrm{H} 2 \mathrm{AX}$, and caveolin-1 were obtained from Cell Signaling Technologies (Danvers, MA) and antibodies to detect flotillin-2 and DHHC5 were obtained from Millipore Sigma (Oakville, ON). Dynasore and desipramine were obtained from Millipore Sigma. Lysine-fixable Alexa488-conjugated dextran (A488-dextran, $10000 \mathrm{MW}$ ), and Alexa-488- phalloidin (A488-phalloidin) were obtained from Thermo Fisher Scientific (Waltham, MA). Latrunculin A was obtained from Cayman Chemical (Ann Arbor, MI).

Cell lines, cell culture and ultrasound treatment. ARPE-19 human retinal pigment epithelial cells (RPE herein) and MDA-MB-231 cells were obtained from American Type Culture Collection (Manassas, VA) and cultured as previously described ${ }^{47}$. Mycoplasma testing was routinely performed by staining with DAPI, approximately every 2 months. Ultrasound treatment was performed on adhered cells as previously described ${ }^{47}$, and as illustrated in Fig. S1. The ultrasound stimulation conditions, activation and treatment with Definity microbubbles (Lantheus Medical Imaging Inc., Saint-Laurent, QC) were previously optimized and characterized; of note, there was $>80 \%$ cell viability upon exposure to USMB stimulation ${ }^{47,64}$.

Inhibitor and drug treatments. For all experiments, some cells were treated with $50 \mu \mathrm{M}$ desipramine for $1 \mathrm{~h}$ prior to USMB treatment. For the dynamin inhibition experiment (Fig. 2E,F), cells were either treated with $80 \mu \mathrm{M}$ dynasore or vehicle control (DMSO) $30 \mathrm{~min}$ prior to USMB treatment. For experiments involving treatment with cisplatin (Figs. 7 and S7A) or doxorubicin (Fig. S7C,D), following treatment with USMB and/ or desipramine, cells were incubated for $2 \mathrm{~h}$ at $37^{\circ} \mathrm{C}$ in growth media containing either $30 \mu \mathrm{M}$ cisplatin or $30 \mu \mathrm{M}$ doxorubicin, after which time the solution containing these drugs was removed, the cells extensively washed to remove any non-internalized drug, followed by incubation at $37 \mathrm{C}$ in regular growth media devoid of any drugs or inhibitors. For experiments involving LatA (Fig. S5), cells were treated with $0.5 \mu \mathrm{M}$ LatA for 30 min prior to additional treatments or fixation.

Plasmid and siRNA transfections. DNA plasmid transfection were performed as previously described using FugeneHD (Promega, Madison, WI) ${ }^{52}$. A DNA plasmid encoding eGFP-DHHC5 was a kind gift from Dr. S. Bamji (University of British Columbia, BC) ${ }^{34}$. This plasmid was used to generate a plasmid encoding eGFP-DHHC5 harbouring a point-mutation corresponding to Y533A by site directed mutagenesis service form BioBasic Inc. (Markham ON). A DNA plasmid encoding flotillin2-eGFP was a kind gift from Dr. G. Fairn (University of Toronto, ON). SiRNA transfections were performed as previously described ${ }^{52}$, with custom siRNA oligonucleotides (Table S1) obtained from Dharmacon (Lafayette, CO).

CRISPR/Cas9 genome editing. CRISPR/Cas9 genome editing to generate MDA-MB-231 lacking functional flotillin-1 was performed using the Edit-R CRISPR/Cas9 system from Dharmacon (Lafayette, CO), as per manufacturer instructions. Briefly, cells were incubated with a mixture of 0.75 pmoles of Edit-R crRNA targeting flotillin-1 (catalog CM-010636-04-0002), 0.75 pmoles of Edit-R tracrRNA (catalog no U-002005-20), $200 \mathrm{ng}$ of Edit-R hCMV-mKate2-Cas9 DNA plasmid and $15 \mu \mathrm{L}$ of Dharmafect Duo (Dharmacon) transfection reagent in $300 \mu \mathrm{L}$ of Opti-MEM media (Thermo Fisher Scientific). Single MDA-MB-231 cells expressing mKate2 were isolated using fluorescence-activated cell sorting; each single cell was grown into separate clonal populations, which were then screened for successful knockout of flotillin-1 by immunoblotting and immunofluorescence microscopy.

Fluorescent dextran fluid-phase internalization assay. RPE or MDA-MB-231 cells grown on coverslips were treated with desipramine for 1 hour prior to USMB treatments. Following USMB stimulation, cells were treated with $10 \mu \mathrm{g} / \mathrm{mL}$ A488-dextran alone or in combination with desipramine at $37 \mathrm{C}$ for $30 \mathrm{~min}$. After the incubation time, cells were extensively washed and immediately fixed in $4 \%$ PFA. Cells were then mounted on glass slides in fluorescence mounting medium (DAKO, Carpinteria, CA).

Immunofluorescence and Alexa 488-phalloidin staining. Endogenous flotillin-1 or -2, DHHC5, caveolin-1 or $\gamma \mathrm{H} 2 \mathrm{AX}$ were labelled by performing indirect immunofluorescence as previously described ${ }^{53}$. Samples were fixed in a solution of $4 \% \mathrm{PFA}$ and permeabilized by Triton-X100 (for flotillin, $\gamma \mathrm{H} 2 \mathrm{AX}$, and caveolin-1 labeling experiments) or ice-cold methanol (for DHHC5 labeling experiments). Alexa 488-phalloidin 
staining (Thermo Fisher Scientific) was performed by treating cells for 60 min with $0.165 \mu \mathrm{M}$ A488-phalloidin following fixation and permeabilization.

Fluorescence microscopy. Widefield epifluorescence microscopy of fixed samples, as shown in Figs. 1, $3 \mathrm{C}, \mathrm{D}, 6 \mathrm{C}, \mathrm{D}, 7 \mathrm{~A}, \mathrm{~B}, \mathrm{D}, \mathrm{F}$ and S2C, S7B,C was performed using a $60 \times(\mathrm{NA} 1.35)$ objective on an Olympus IX83 epifluorescence microscope using a Hamamatsu ORCA FLASH4.0 C11440-22CU camera. Images were acquired using cellSens software (Olympus, Canada, Richmond Hill, ON).

Spinning disk confocal and TIRF microscopy of fixed samples was performed using a Quorum (Guelph, ON, Canada) Diskovery combination TIRF and spinning-disc confocal microscope. This instrument is comprised of a Leica DMi8 microscope equipped with a $63 \times / 1.49$ NA objective with a $1.8 \times$ camera relay (total magnification $108 \times)$. Imaging was done using 488, $561 \mathrm{~nm}$ laser illumination and 527/30, 630/75 emission filters and acquired using a Zyla 4.2Plus sCMOS camera (Hamamatsu, Bridgewater, NJ). TIRF microscopy imaging of live cells was performed similarly while maintaining cells in temperature $(37 \mathrm{C})$ and $\mathrm{CO}_{2}$-controlled stage. Time-lapse image series were acquired starting at $5 \mathrm{~min}$ after USMB treatment, time lapse images were taken every $60 \mathrm{~s}$ for $15 \mathrm{~min}$.

Image analysis. Quantification of whole-cell intensity of flotillin-1, caveolin-1, DHHC5, or internalized fluid-phase A488-dextran, or nuclear $\gamma \mathrm{H} 2 \mathrm{AX}$ was performed as previously described ${ }^{52,53}$ using ImageJ software (National Institutes of Health, Bethesda, MD) ${ }^{65}$. Detection and analysis of diffraction-limited internalized flotillin-1 puncta (Fig. 2G,H), was performed using custom software developed in Matlab (Mathworks Corporation, Natick, MA), as described previously for cell surface endocytic structures ${ }^{48,66-68}$ and internalized vesicles or endosomes ${ }^{52}$. Detection, tracking and analysis of cell surface flotillin2-eGFP in time-lapse image series obtained by TIRF-M (Fig. 2B-D) was performed as previously described for cell surface clathrin structures ${ }^{48,69}$.

Immunoblotting. Whole cell lysates were prepared in Laemmli sample buffer, resolved by SDS-PAGE and probed with specific antibodies, and the resulting signal intensity quantification were performed as previously described $^{53}$. To examine phosphorylation of DHHC5 (Fig. 4A), we used the phos-tag gel system, which results in exaggeration of differences in apparent molecular weight of phosphorylated forms of specific proteins, as previously described ${ }^{53}$.

Cell viability measurements. To perform the proliferation/viability assay as shown in Fig. 7B, 24h following treatments as indicated cells were detached using $0.025 \%$ trypsin and cells were counted using a Countess II FL Automated Cell Counter (Thermo Fisher Scientific). The number of non-viable cells was determined by the loss of cells from the initial number of seeded cells and expressed as a normalized value. To perform the cell proliferation/viability assay as shown in Fig. S7A, RPE cells seeded in 6-well plates were treated as indicated. After $24 \mathrm{~h}$, cells were stained with crystal violet, then solubilized in a solution of $0.1 \%$ SDS, followed by measurement of absorbance at $595 \mathrm{~nm}$. Cell viability was expressed as percentage of the crystal violet signal in control (untreated) cells.

Statistical analyses. Statistical analysis was performed as previously described ${ }^{53}$. Measurements of samples involving two conditions (Figs. S2A, S3, S4A, S5 and S6) were analyzed by student's t-test, with p $<0.05$ as a threshold for statistically significant difference between conditions. Measurements of samples involving one experimental parameter and more than two conditions (Figs. 2A, B,H and S6A, S6C) were analyzed by one-way ANOVA, followed by Tukey post-test to compare differences between conditions, with $\mathrm{p}<0.05$ as a threshold for statistically significant difference between conditions. Measurements of samples involving two experimental parameters (Figs. 1B, 2F, 3B,D, 4C, 5B, 6B,D and 7B,C,E) were analyzed by two-way ANOVA, followed by Tukey post-test to compare differences between conditions, with $\mathrm{p}<0.05$ as a threshold for statistically significant difference between conditions.

Received: 11 February 2019; Accepted: 7 November 2019;

Published online: 28 November 2019

\section{References}

1. Tiwari, G. et al. Drug delivery systems: An updated review. Int. J. Pharm. Investig. 2, 2 (2012).

2. Wang, D. \& Lippard, S. J. Cellular processing of platinum anticancer drugs. Nat. Rev. Drug Discov. 4, 307-20 (2005).

3. Yang, N. \& Hinner, M. Getting Across the Cell Membrane: An Overview for Small Molecules, Peptides, and Proteins. Methods Mol Biol. 1266, 29-53 (2015).

4. Florea, A.-M., Büsselberg, D., Florea, A.-M. \& Büsselberg, D. Cisplatin as an Anti-Tumor Drug: Cellular Mechanisms of Activity, Drug Resistance and Induced Side Effects. Cancers (Basel). 3, 1351-1371 (2011).

5. Dasari, S. \& Bernard Tchounwou, P. Cisplatin in cancer therapy: Molecular mechanisms of action. Eur. J. Pharmacol. 740, 364-378 (2014).

6. Swaan, L. M. B. \& Swaan, P. W. Endocytic Mechanisms for Targeted Drug Delivery Lisa. Adv. Drug Deliv. Rev. 59, 748-758 (2007).

7. Akinc, A. \& Battaglia, G. Exploiting endocytosis for nanomedicines. Cold Spring Harb. Perspect. Biol. 5, a016980 (2013).

8. McMahon, H. T. \& Boucrot, E. Molecular mechanism and physiological functions of clathrin-mediated endocytosis. Nat. Rev. Mol. Cell Biol. 12, 517-33 (2011).

9. Mayor, S., Parton, R. G. \& Donaldson, J. G. Clathrin-independent pathways of endocytosis. Cold Spring Harb. Perspect. Biol. 6, a016758 (2014).

10. Glebov, O. O., Bright, N. A. \& Nichols, B. J. Flotillin-1 defines a clathrin-independent endocytic pathway in mammalian cells. Nat Cell Biol 8, 46-54 (2006).

11. Ferreira, A. P. A. \& Boucrot, E. Mechanisms of Carrier Formation during Clathrin-Independent Endocytosis. Trends Cell Biol. 28, $188-200(2018)$ 
12. Lang, D. M. et al. Identification of reggie-1 and reggie- 2 as plasmamembrane-associated proteins which cocluster with activated GPI-anchored cell adhesion molecules in non-caveolar micropatches in neurons. J Neurobiol 37, 502-523 (1998).

13. Neumann-Giesen, C., Fernow, I., Amaddii, M. \& Tikkanen, R. Role of EGF-induced tyrosine phosphorylation of reggie-1/flotillin-2 in cell spreading and signaling to the actin cytoskeleton. J. Cell Sci. 120, 395-406 (2007).

14. Rivera-Milla, E., Stuermer, C. A. O. \& Málaga-Trillo, E. Ancient origin of reggie (flotillin), reggie-like, and other lipid-raft proteins: Convergent evolution of the SPFH domain. Cell. Mol. Life Sci. 63, 343-357 (2006).

15. Neumann-Giesen, C. et al. Membrane and raft association of reggie-1/flotillin-2: role of myristoylation, palmitoylation and oligomerization and induction of filopodia by overexpression. Biochem J 378, 509-518 (2004).

16. Babuke, T. et al. Hetero-oligomerization of reggie-1/flotillin-2 and reggie-2/flotillin-1 is required for their endocytosis. Cell. Signal. 21, 1287-1297 (2009).

17. Li, Y., Martin, B. R., Cravatt, B. F. \& Hofmann, S. L. DHHC5 protein palmitoylates flotillin-2 and is rapidly degraded on induction of neuronal differentiation in cultured cells. J. Biol. Chem. 287, 523-30 (2012).

18. Slaughter, N. et al. The flotillins are integral membrane proteins in lipid rafts that contain TCR-associated signaling components: implications for T-cell activation. Clin. Immunol. 584, 2740-2747 (2010).

19. Meister, M. \& Tikkanen, R. Endocytic trafficking of membrane-bound cargo: a flotillin point of view. Membranes (Basel). 4, 356-71 (2014).

20. Blanchet, M. H. et al. Cripto recruits Furin and PACE4 and controls Nodal trafficking during proteolytic maturation. EMBO J. 27, 2580-2591 (2008).

21. Otto, G. P. \& Nichols, B. J. The roles of flotillin microdomains - endocytosis and beyond. J. Cell Sci. 124, 3933-3940 (2011).

22. Payne, C. K., Jones, S. A., Chen, C. \& Zhuang, X. Internalization and Trafficking of Cell Surface Proteoglycans and ProteoglycanBinding Ligands. Traffic 8, 389-401 (2007).

23. Ge, L. et al. Flotillins play an essential role in Niemann-Pick C1-like 1-mediated cholesterol uptake. Proc. Natl. Acad. Sci. 108, 551-556 (2011).

24. Riento, K., Frick, M., Schafer, I. \& Nichols, B. J. Endocytosis of flotillin-1 and flotillin-2 is regulated by Fyn kinase. J. Cell Sci. 122, 912-8 (2009)

25. Lariccia, V. et al. Massive calcium-activated endocytosis without involvement of classical endocytic proteins. J. Gen. Physiol. 137, $111-132(2011)$

26. Hilgemann, D. W., Fine, M., Linder, M. E., Jennings, B. C. \& Lin, M.-J. Massive endocytosis triggered by surface membrane palmitoylation under mitochondrial control in BHK fibroblasts. Elife 2, e01293 (2013).

27. Hilgemann, D. W. \& Fine, M. Mechanistic analysis of massive endocytosis in relation to functionally defined surface membrane domains. J. Gen. Physiol. 137, 155-72 (2011).

28. Mitchell, D. A., Vasudevan, A., Linder, M. E. \& Deschenes, R. J. Thematic review series: Lipid Posttranslational Modifications. Protein palmitoylation by a family of DHHC protein S -acyltransferases: Fig. 1. J. Lipid Res. 47, 1118-1127 (2006).

29. Rocks, O. et al. The palmitoylation machinery is a spatially organizing system for peripheral membrane proteins. Cell 141, 458-471 (2010).

30. Tabaczar, S., Czogalla, A., Podkalicka, J., Biernatowska, A. \& Sikorski, A. F. Protein palmitoylation: Palmitoyltransferases and their specificity. Exp. Biol. Med. 242, 1150-1157 (2017).

31. El-Husseini, A. E. D. et al. Synaptic strength regulated by palmitate cycling on PSD-95. Cell 108, 849-863 (2002).

32. Kaur, I. et al. Activity-Dependent Palmitoylation Controls SynDIG1 Stability, Localization, and Function. J. Neurosci. 36, 7562-7568 (2016).

33. Thomas, G. M. et al. by DHHC5/8 targets GRIP1 to dendritic endosomes to regulate AMPA-R trafficking. Magn Reson Imaging 31, 477-479 (2013).

34. Brigidi, G. S., Santyr, B., Shimell, J., Jovellar, B. \& Bamji, S. X. Activity-regulated trafficking of the palmitoyl-acyl transferase DHHC5. Nat. Commun. 6, 1-17 (2015).

35. Kiessling, F., Fokong, S., Koczera, P., Lederle, W. \& Lammers, T. Ultrasound microbubbles for molecular diagnosis, therapy, and theranostics. J. Nucl. Med. 53, 345-8 (2012).

36. Chen, H. \& Hwang, J. H. Ultrasound-targeted microbubble destruction for chemotherapeutic drug delivery to solid tumors. J. Ther. ultrasound 1,10 (2013).

37. Todorova, M. et al. Antitumor effects of combining metronomic chemotherapy with the antivascular action of ultrasound stimulated microbubbles. Int. J. Cancer 132, 2956-2966 (2013).

38. Tsutsui, J. M., Xie, F. \& Porter, R. T. The use of microbubbles to target drug delivery. Cardiovasc. Ultrasound 2, 1-7 (2004)

39. Wang, T.-Y., Wilson, K., Machtaler, S. \& Willmann, J. Ultrasound and Microbubble Guided Drug Delivery: Mechanistic Understanding and Clinical Implications. Curr. Pharm. Biotechnol. 14, 743-752 (2014).

40. Dijkmans, P. A. et al. Microbubbles and ultrasound: from diagnosis to therapy, https://doi.org/10.1016/j.euje.2004.02.001 (2004).

41. Sirsi, S. \& Borden, M. Microbubble Compositions, Properties and Biomedical Applications. Bubble Sci. Eng. Technol. 1, 3-17 (2009).

42. Ferrara, K., Pollard, R. \& Borden, M. Ultrasound Microbubble Contrast Agents: Fundamentals and Application to Gene and Drug Delivery. Annu. Rev. Biomed. Eng. 9, 415-447 (2007).

43. De Cock, I. et al. Ultrasound and microbubble mediated drug delivery: acoustic pressure as determinant for uptake via membrane pores or endocytosis. J. Control. Release 197, 20-8 (2015)

44. Hu, Y., Wan, J. M. F. \& Yu, A. C. H. Membrane Perforation and Recovery Dynamics in Microbubble-Mediated Sonoporation. Ultrasound Med. Biol. 39, 2393-2405 (2013).

45. Tardoski, S. et al. Low-Intensity Ultrasound Promotes Clathrin-Dependent Endocytosis for Drug Penetration into Tumor Cells. Ultrasound Med. Biol. 41, 2740-54 (2015).

46. Sennoga, C. A. et al. Microbubble-mediated ultrasound drug-delivery and therapeutic monitoring. Expert Opin. Drug Deliv. 14, 1031-1043 (2017).

47. Fekri, F., Delos Santos, R., Karshafian, R. \& Antonescu, C. Ultrasound microbubble treatment enhances clathrin-mediated endocytosis and fluid-phase uptake through distinct mechanisms. PLoS One 11, e0156754 (2016).

48. Aguet, F., Antonescu, C. N., Mettlen, M., Schmid, S. L. \& Danuser, G. Advances in analysis of low signal-to-noise images link dynamin and AP2 to the functions of an endocytic checkpoint. Dev. Cell 26, 279-91 (2013).

49. Kurrle, N., John, B., Meister, M. \& Tikkanen, R. Function of flotillins in receptor tyrosine kinase signaling and endocytosis: role of tyrosine phosphorylation and oligomerization. Protein phosphorylation Hum. Heal, https://doi.org/10.5772/48598 (2012).

50. Macia, E. et al. Dynasore, a cell-permeable inhibitor of dynamin. Dev Cell 10, 839-850 (2006).

51. Park, R. J. et al. Dynamin triple knockout cells reveal off target effects of commonly used dynamin inhibitors. J. Cell Sci. 126, 5305-12 (2013).

52. Bone, L. N. et al. The acyltransferase LYCAT controls specific phosphoinositides and related membrane traffic. Mol. Biol. Cell 28, 161-172 (2017).

53. Bautista, S. J. et al. mTOR complex 1 controls the nuclear localization and function of glycogen synthase kinase 33. J. Biol. Chem. 293, 14723-14739 (2018).

54. Cai, C. \& Chen, J. Overexpression of caveolin-1 induces alteration of multidrug resistance in Hs578T breast adenocarcinoma cells. Int. J. Cancer 111, 522-529 (2004)

55. Lee, C. Y. et al. The influence of a caveolin-1 mutant on the function of P-glycoprotein. Sci. Rep. 6, 20486 (2016). 
56. Jang, D., Kwon, H., Jeong, K., Lee, J. \& Pak, Y. Essential role of flotillin-1 palmitoylation in the intracellular localization and signaling function of IGF-1 receptor. J. Cell Sci. 128, 2179-90 (2015).

57. Clingen, P. H. et al. Histone H2AX phosphorylation as a molecular pharmacological marker for DNA interstrand crosslink cancer chemotherapy. Biochem. Pharmacol. 76, 19-27 (2008).

58. Liu, J., DeYoung, S. M., Zhang, M., Dold, L. H. \& Saltiel, A. R. The stomatin/prohibitin/flotillin/HflK/C domain of flotillin-1 contains distinct sequences that direct plasma membrane localization and protein interactions in 3T3-L1 adipocytes. J. Biol. Chem. 280, $16125-16134$ (2005).

59. Morrow, I. C. et al. Flotillin-1/reggie-2 traffics to surface raft domains via a novel Golgi-independent pathway. Identification of a novel membrane targeting domain and a role for palmitoylation. J. Biol. Chem. 277, 48834-48841 (2002).

60. Dimcevski, G. et al. A human clinical trial using ultrasound and microbubbles to enhance gemcitabine treatment of inoperable pancreatic cancer. J. Control. Release 243, 172-181 (2016).

61. Sorace, A. G., Warram, J. M., Umphrey, H. \& Hoyt, K. Microbubble-mediated ultrasonic techniques for improved chemotherapeutic delivery in cancer. J. Drug Target. 20, 43-54 (2012).

62. Kato, S., Shirai, Y., Sakamoto, M., Mori, S. \& Kodama, T. Use of a Lymphatic Drug Delivery System and Sonoporation to Target Malignant Metastatic Breast Cancer Cells Proliferating in the Marginal Sinuses. Sci. Rep. 9, 13242 (2019).

63. Goertz, D. E. et al. Antitumor Effects of Combining Docetaxel (Taxotere) with the Antivascular Action of Ultrasound Stimulated Microbubbles. PLoS One 7, e52307 (2012).

64. Karshafian, R., Bevan, P. D., Williams, R., Samac, S. \& Burns, P. N. Sonoporation by Ultrasound-Activated Microbubble Contrast Agents: Effect of Acoustic Exposure Parameters on Cell Membrane Permeability and Cell Viability. Ultrasound Med. Biol. 35, 847-860 (2009).

65. Schneider, C. A., Rasband, W. S. \& Eliceiri, K. W. NIH Image to ImageJ: 25 years of image analysis. Nat. Methods 9, 671-5 (2012).

66. Garay, C. et al. Epidermal growth factor-stimulated Akt phosphorylation requires clathrin or ErbB2 but not receptor endocytosis. Mol. Biol. Cell 26, 3504-19 (2015).

67. Delos Santos, R. C. et al. Selective regulation of clathrin-mediated epidermal growth factor receptor signaling and endocytosis by phospholipase C and calcium. Mol. Biol. Cell 28 (2017).

68. Lucarelli, S., Delos Santos, R. C. \& Antonescu, C. N. Measurement of epidermal growth factor receptor-derived signals within plasma membrane clathrin structures. Methods in Molecular Biology 1652, 191-225 (2017).

69. Jaqaman, K. et al. Robust single-particle tracking in live-cell time-lapse sequences. Nat. Methods 5, 695-702 (2008)

\section{Acknowledgements}

Funding for this research was provided by a Discovery Grant from the Natural Sciences and Engineering Research Council, a New Investigator Award from the Canadian Institutes of Health Research (CIHR) and an Early Researcher Award (Ontario Ministry of Research, Innovation and Science) to C.N.A., a Discovery Grant from the Natural Sciences and Engineering Research Council to R.K. and an Ontario Graduate Scholarship to F.F. and S.B.

\section{Author contributions}

F.F., C.N.A. and R.K. wrote the main manuscript text, F.F., J.A., S.B., L.O. and R.M.D. performed the experiments, and F.F., J.A. and C.N.A. prepared the figures. All authors reviewed the manuscript.

\section{Competing interests}

The authors declare no competing interests.

\section{Additional information}

Supplementary information is available for this paper at https://doi.org/10.1038/s41598-019-54062-9.

Correspondence and requests for materials should be addressed to C.N.A. or R.K.

Reprints and permissions information is available at www.nature.com/reprints.

Publisher's note Springer Nature remains neutral with regard to jurisdictional claims in published maps and institutional affiliations.

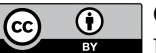

Open Access This article is licensed under a Creative Commons Attribution 4.0 International

License, which permits use, sharing, adaptation, distribution and reproduction in any medium or format, as long as you give appropriate credit to the original author(s) and the source, provide a link to the Creative Commons license, and indicate if changes were made. The images or other third party material in this article are included in the article's Creative Commons license, unless indicated otherwise in a credit line to the material. If material is not included in the article's Creative Commons license and your intended use is not permitted by statutory regulation or exceeds the permitted use, you will need to obtain permission directly from the copyright holder. To view a copy of this license, visit http://creativecommons.org/licenses/by/4.0/.

(c) The Author(s) 2019 